\title{
Desbaste seletivo em povoamentos de Tectona grandis com diferentes idades
}

\author{
Sidney Fernando CALDEIRA¹, Daniel Leandro Costa OLIVEIRA². \\ RESUMO \\ O efeito da intensidade do primeiro desbaste seletivo, após um ano, foi avaliado em dois povoamentos de teca sob espaçamento \\ 3,0 × 3,0 m, e em duas diferentes idades. No povoamento com quatro anos de idade, o desbaste foi de $20 \%$, 30\% e 40\%, \\ em número de indivíduos, e no de cinco anos, de 30\%, 40\% e 50\%, e as respectivas testemunhas (0\%). Cada tratamento \\ foi aplicado ao acaso, com oito repetições, contendo cada parcela 35 árvores, além da bordadura. Um ano após o desbaste \\ aplicado no povoamento com quatro anos, as variáveis DAP, altura total $(\mathrm{H})$ e as médias por árvore de área transversal $(\bar{g})$ e \\ de volume $(\bar{V})$ não apresentaram diferenças significativas entre os tratamentos; o incremento corrente anual (ICA $\left.{ }_{V}\right)$ não foi \\ afetado pelas intensidades. No povoamento desbastado aos cinco anos, o ICA $\mathrm{I}_{V} \mathrm{e} \mathrm{H}$ foram semelhantes em todos tratamentos. \\ As intensidade de desbaste testadas não afetaram o crescimento em DAP, $\bar{g}$ e $\bar{V}$, mas apresentaram diferenças significativas \\ em relação à testemunha. O primeiro desbaste seletivo pode ser aplicado aos cinco anos de idade, em povoamentos de Tectona \\ grandis com condiçôes semelhantes, com qualquer das intensidades testadas.
}

PALAVRAS-ChaVE: Intensidade de desbaste, Primeiro desbaste seletivo, Reflorestamento, Teca.

\section{Selective thinning in Tectona grandis plantations with different ages}

\section{ABSTRACT}

The intensity of the first selective thinning was evaluated in two plantations, $3.0 \times 3.0 \mathrm{~m}$. In the four year old plantation, the thinning was $20 \%, 30 \%$ and $40 \%$ in density and in the five year old, it was 30\%, $40 \%$ and 50\%, and the respective controls $(0 \%)$. Each treatment was applied in eight random blocks of 35 trees, beyond the border. One year after thinning at the age of four, the $\mathrm{DBH}$, total height $(\mathrm{H})$, and average by tree of basal area $(\bar{g})$ and of volume $(\bar{V})$ presented no differences among the treatments; the current annual increment $\left(\mathrm{CAI}_{V}\right)$ was not affected by the intensities. In the plantation thinned at the age of five, the $\mathrm{CAI}_{V}$ and $\mathrm{H}$ were similar in all treatments; the $\mathrm{DBH}, \bar{g}$ and $\bar{V}$ were not affected by the tested intensities, but they were greater than the control. The first selective thinning may be applied at the age of five, in Tectona grandis plantations with similar conditions, at any tested intensity.

KEYWORDS: First selective thinning, Reforestation, Teak, Thinning intensities.

1 Universidade Federal de Mato Grosso, Pró-Reitoria de Ensino e Graduação, Departamento de Engenharia Florestal. Av. Fernando Correa da Costa s/n Coxipó. CEP $78068-000$ Cuiabá - MT - Brasil. Telefone: (65) 36158659 Fax: (65) 3615 8631. e-mail: sidcal@ufmt.br

2 Vila Militar I, Ap. 203, Bom Clima. 78195-300, Chapada dos Guimarães-MT. e-mail: dlcoflorestal@yahoo.com.br 


\section{INTRODUÇÃO}

A teca, Tectona grandis, da família Lamiaceae, antes incluída em Verbenaceae, é uma espécie exótica que se adaptou bem no estado de Mato Grosso, onde os primeiros plantios comerciais foram feitos no município de Cáceres, a partir de 1970. Atualmente a área plantada neste Estado é estimada em mais de 50 mil hectares.

Ainda que existam várias opções para o espaçamento inicial, as altas densidades podem afetar o crescimento em diâmetro, a altura e a produtividade (Salas \& Gonzáles, 2003), principalmente sem um adequado programa de desbaste, quanto ao tipo, época de aplicação, e a intensidade da operação.

De outro lado, o plantio de 1000 a 2000 árvores.ha ${ }^{-1}$ permite uma adequada seleção nos desbastes (Pandey \& Brown, 2000), e o uso de espaçamentos menores com desbastes freqüentes parecem ser mais promissores (Jerez et al., 2003).

Segundo Figueiredo (2001), no Acre, os plantios com 1666 a 2000 árvores por hectare, equivalentes a espaçamentos de $2,3 \times 2,0 \mathrm{~m}$ a $3,0 \times 2,0 \mathrm{~m}$, nos sítios superiores, apresentaram o melhor desempenho silvicultural.

A idade de aplicação do primeiro desbaste depende da qualidade de sítio, e pode ser efetuado, dos três aos seis anos de idade, quando as árvores alcançam uma altura média de 8,0 m (Chaves \& Fonseca, 1991) ou de 9,0 a 9,5 m (Krishnapillay, 2000).

Em povoamentos tropicais, segundo Galloway et al. (2001), o fechamento do dossel tem sido utilizado como um bom indicador para a época de aplicação do primeiro desbaste, pois tem correlação com a redução do crescimento em diâmetro.

Existem indicações mais específicas, quanto à idade e à intensidade de aplicação do primeiro desbaste, em teca: aos quatro anos e remoção de $40 \%$ das árvores ou então remoção de $25 \%$ das árvores seguida de nova retirada ao quinto ano do mesmo número de árvores (Cordero \& Kanninen, 2003); ou a remoção de 40 a $60 \%$ das árvores sem as características desejáveis (Briscoe, 1995); e até para desbastes tardios, em povoamentos $4,0 \times 4,0 \mathrm{~m}$, a retirada de até $60 \% \mathrm{em}$ densidade, não oferece danos ao povoamento, e entre $10 \mathrm{e}$ 14 anos de idade, é aplicado o desbaste comercial (Vincent et al., 2000).

As diferenças no crescimento e desenvolvimento e a falta de informações sobre desbaste em teca no Brasil motivaram este estudo para verificar o efeito da intensidade do primeiro desbaste em povoamentos dessa espécie, com diferentes idades.

\section{MATERIAL E MÉTODOS}

No município de Nossa Senhora do Livramento (16 12' $32^{\prime \prime} \mathrm{S}$ e $\left.56^{\circ} 22^{\prime} 57^{\prime \prime} \mathrm{W}\right)$, os plantios de teca foram iniciados em 1999, no espaçamento 3,0 x 3,0 m e 3,0 x 2,0 m. O clima da região apresenta a seguinte característica, em valores médios: precipitação de $1300 \mathrm{~mm}$. ano ${ }^{-1}$; temperatura anual de $25^{\circ} \mathrm{C}$, com mínimas de $20^{\circ} \mathrm{C}$ e máximas de $32^{\circ} \mathrm{C}$; umidade relativa de $76 \%$ e evapotranspiração potencial de $4,1 \mathrm{~mm} \cdot \mathrm{dia}^{-1}$.

Em julho de 2004, parcelas experimentais foram estabelecidas em dois povoamentos de teca, um com quatro e outro com cinco anos de idade, cujas características de crescimento e desenvolvimento (Caldeira \& Borges, 2004) são apresentadas na Tabela 1.

Amostras coletadas nas parcelas experimentais revelaram que o Planossolo Eutrófico da área apresenta teores médios de areia, silte e argila, respectivamente de 48,5; 11,5 e 40\%, e as características químicas: de 6,00 a 6,30 unidades de $\mathrm{pH}$; teores de Fósforo e Potássio, respectivamente, entre 19,1 a 19,5 e 101 a 139 mg.dm³ ${ }^{-3}$, Cálcio + Magnésio de 4,9 a 6,1 $\mathrm{cmol}_{c} \cdot \mathrm{dm}^{-3}$, Cálcio entre 3,4 a $5,0 \mathrm{cmol}_{c} \cdot \mathrm{dm}^{-3}$, sem a presença de Alumínio; e matéria orgânica entre 1,4 a 1,9\%.

Em agosto de 2004, de forma casualizada, cada tratamento foi repetido em oito parcelas de 21,0 x 15,0 m, cada uma com 35 árvores, mais a bordadura. Os tratamentos foram desbastes seletivos, nas intensidades de 20\%, 30\% e 40\%, em número de indivíduos, para o povoamento com quatro anos de idade, e de $30 \%, 40 \%$ e $50 \%$ para o povoamento com cinco anos e as respectivas testemunhas.

Os critérios para a seleção das árvores a serem desbastadas foram, em ordem cronológica: estado fitossanitário, forma e qualidade do fuste, e as árvores com menores valores de diâmetro à altura do peito e de altura total do fuste.

Foram registrados o DAP e altura total de cada árvore e calculados a área basal e o volume total (Matricardi, 1989), e as médias, por árvore de área transversal e de volume total com casca, antes da aplicação dos tratamentos, em cada povoamento. Em setembro de 2005, foram registradas e

Tabela 1 - Idade, espaçamento inicial (EI), densidade (D), DAP, altura total $(\mathrm{H})$, área basal $(\mathrm{G})$ e área transversal por árvore $(\mathrm{gm})$ de dois povoamentos de Tectona grandis, submetidos a desbaste seletivo, Nossa Senhora do Livramento, MT, Brasil.

\begin{tabular}{cccccccc}
\hline $\begin{array}{c}\text { Ano } \\
\text { de } \\
\text { plantio }\end{array}$ & $\begin{array}{c}\text { Idade } \\
(\text { anos })\end{array}$ & $\begin{array}{c}\text { EI } \\
(\mathrm{m})\end{array}$ & $\begin{array}{c}\text { D } \\
\left(\text { árvores.ha }{ }^{-1}\right)\end{array}$ & $\begin{array}{c}\text { DAP } \\
(\mathrm{cm})\end{array}$ & $\begin{array}{c}\mathbf{H} \\
(\mathrm{m})\end{array}$ & $\begin{array}{c}\mathbf{G} \\
\left(\mathrm{m}^{2} . \mathrm{ha}^{-1}\right)\end{array}$ & $\begin{array}{c}\text { gm } \\
\left(\mathrm{m}^{2} . \text { árvore }^{-1}\right)\end{array}$ \\
\hline 1999 & 5 & $3,0 \times 3,0$ & 1083 & 12,3 & 10,4 & 12,9875 & 0,0120 \\
2000 & 4 & $3,0 \times 3,0$ & 1088 & 9,5 & 9,1 & 7,7611 & 0,0071 \\
\hline
\end{tabular}


calculadas as mesmas variáveis das árvores remanescentes, e o incremento corrente anual do volume.

De forma independente para cada povoamento, após o teste de normalidade, os valores das variáveis foram submetidos à análise de variância e as médias de cada tratamento comparadas pelo teste de Skott-Knott, a 5\%, com o software SISVAR 5.0 (Ferreira, 2000).

\section{RESULTADOS E DISCUSSÃO}

$\mathrm{Na}$ Tabela 2 estão apresentadas as variáveis registradas aos cinco anos de idade, para o povoamento 3,0 x 3,0 m, desbastado no ano anterior nas intensidades de 20\%, 30\% e $40 \%$. O DAP, a altura total e as médias de área transversal e volume por árvore não foram afetados, provavelmente pela disponibilidade de espaço para crescimento, tanto na parte aérea como no solo. Por isso, no ano seguinte ao tratamento, as árvores da testemunha não sofreram os efeitos da competição ou este efeito foi muito pequeno.

Ainda assim, os valores individuais de DAP e as médias por árvore de área transversal e de volume, na testemunha, foram menores, em relação aos valores dos tratamentos, o que indica o início da competição intraespecífica.

Como era de se esperar, os tratamentos afetaram a área basal e o volume, cujos valores foram inferiores ao da testemunha, e isto ocorreu em função da densidade. Ainda assim, os tratamentos com 20 e 30\% em intensidade de desbaste não diferiram entre si e se observou uma diminuição gradativa nestes valores com o aumento da intensidade, também como conseqüência da densidade pós-desbaste.

O ICA foi afetado pelos tratamentos, mas, nesse caso, somente a testemunha foi superior às intensidades testadas e estas não diferiram entre si. Isso pode ser atribuído à diferença de $20 \%$ em densidade, comparando-se a testemunha para a menor intensidade utilizada, enquanto a diferença entre as intensidades foi de apenas $10 \%$. Assim, o período de um ano pode não ter sido suficiente para que o incremento das árvores tratadas fosse maior que o da testemunha.
Um ano após a operação, o desbaste aplicado aos quatro anos de idade não apresentou efeitos nas variáveis individuais analisadas, pois havia espaço disponível. As diferenças das variáveis relacionadas com o povoamento foram decorrentes das diferenças de densidade. Segundo Pérez \& Kanninen (2005), o desbaste aos quatro anos somente é indicado para sítios de alta qualidade, a 50\%, para obtenção do máximo de diâmetro em rotação de 30 anos.

Cordero \& Kanninen (2003) registraram aumento de até $5,0 \mathrm{~cm}$ no DAP, dois anos após a aplicação de desbaste a $40 \%$ em densidade, aos quatro anos, em relação à testemunha, além da recuperação da área basal extraída, de melhor forma do que o desbaste executado aos seis anos.

$\mathrm{Na}$ Tabela 3 estão as variáveis registradas aos seis anos de idade, para o povoamento $3,0 \times 3,0 \mathrm{~m}$, desbastado aos cinco anos e que resultaram em diferenças significativas entre a testemunha e os tratamentos para as variáveis individuais (DAP, H, $\bar{g}$ e $\bar{V}$ ), com valores menores para a testemunha em relação às áreas desbastadas, e estas sem diferenças entre si.

Salas \& Gonzáles (2003) encontraram incremento em diâmetro semelhante, contudo, o resultado foi obtido com a aplicação de desbaste em povoamento de teca com seis anos de idade e uma densidade remanescente de apenas 500 árvores.

No povoamento desbastado aos cinco anos de idade, as variáveis área basal e o volume remanescentes apresentaram diferenças significativas entre todos os tratamentos e os maiores valores $\left(19,8049 \mathrm{~m}^{2} \cdot \mathrm{ha}^{-1}\right.$ e 140,6208 $\left.\mathrm{m}^{3} \cdot \mathrm{ha}^{-1}\right)$ foram registrados para a testemunha, com diminuição gradativa nos tratamentos, de acordo com o aumento da intensidade de desbaste. Isto indica que o efeito da remoção das árvores no ano anterior ainda é maior do que o efeito que a liberação de espaço pode propiciar para as árvores remanescentes.

A altura total e o ICAv não foram afetados pelos tratamentos, indicando que a liberação de espaço no primeiro ano, foi suficiente para o desenvolvimento das árvores

Tabela 2 - Densidade (D), área basal (G), volume total (V), ICA, DAP, altura (H), e médias, por árvore, de área transversal (gm) e de volume (vm) de Tectona grandis, aos cinco anos de idade, remanescentes de desbaste seletivo aplicado no ano anterior, nas intensidades de 20\%, 30\% e $40 \%$ em densidade (D20, D30 e D40) e da testemunha (T00), em um povoamento implantado a 3,0 x 3,0 m, Nossa Senhora do Livramento, MT. Médias de cada variável seguidas da mesma letra não diferem entre si pelo teste de Skott-Knott a $5 \%$.

\begin{tabular}{|c|c|c|c|c|c|c|c|c|c|c|c|c|c|}
\hline Intensidade & D & $\mathbf{G}$ & & V & & ICAv & & DAP & & H & & gm & vm \\
\hline (\%) & $($ árvores.ha-1) & $\left(m^{2} . h^{-1}\right)$ & & $\left(m^{3} \cdot h a^{-1}\right)$ & & $\left(\mathrm{m}^{3} \cdot \mathrm{ha}^{-1} \cdot \mathrm{an}\right.$ & & $(\mathrm{cm})$ & & $(\mathrm{m})$ & & $\left(m^{2}\right.$.árvore-1) & $\left(m^{3}\right.$.árvore $\left.{ }^{-1}\right)$ \\
\hline TOO & 1111 & 17,1196 & $A$ & 110,1034 & A & 35,9999 & A & 13,9 & A & 12,7 & A & $0,0154 \quad \mathrm{~A}$ & $0,0991 \mathrm{~A}$ \\
\hline D20 & 871 & 14,2863 & B & 91,8335 & B & 30,3073 & B & 14,4 & A & 12,6 & A & $0,0165 \quad A$ & $0,1064 \quad A$ \\
\hline D30 & 769 & 13,1733 & B & 86,4659 & B & 28,5563 & B & 14,5 & A & 12,7 & A & $0,0172 \quad A$ & 0,1127 \\
\hline D40 & 658 & 11,5264 & C & 74,4019 & C & 29,1598 & B & 14,8 & A & 12,6 & A & $0,0176 \quad A$ & $0,1136 \quad A$ \\
\hline CV (\%) & 5,9 & 7,9 & & 8,9 & & 19,0 & & 4,8 & & 4,5 & & 9,4 & 11,2 \\
\hline Erro padrão & 17 & 0,3953 & & 2,8535 & & 2,0883 & & 0,2 & & 0,2 & & 0,0006 & 0,0043 \\
\hline
\end{tabular}


Tabela 3 - Densidade (D), área basal (G), volume total (V), ICA, DAP, altura (H) e médias individuais de área transversal (Gm) e de volume (Vm) de Tectona grandis, aos seis anos de idade, remanescentes de desbaste seletivo aplicado no ano anterior, nas intensidades de 30\%, 40\% e 50\% em densidade (D30, D40 e D50) e da testemunha (T00), em um povoamento implantado a 3,0 x 3,0 m, Nossa Senhora do Livramento, MT. Médias de cada variável seguidas da mesma letra não diferem entre si pelo teste de Skott-Knott a $5 \%$.

\begin{tabular}{|c|c|c|c|c|c|c|c|c|c|c|}
\hline Intensidade & D & G & & V & & ICAv & DAP & H & $\mathrm{gm}$ & vm \\
\hline (\%) & (árvores.ha-1) & $\left(m^{2} \cdot h^{-1}\right)$ & & $\left(m^{3} \cdot h a^{-1}\right)$ & & $\left(\mathrm{m}^{3} \cdot \mathrm{ha}^{-1} \cdot \mathrm{ano}\right)$ & (cm) & (m) & $\left(\mathrm{m}^{2}\right.$.árvore $\left.{ }^{-1}\right)$ & $\left(\mathrm{m}^{3}\right.$. árvore $\left.{ }^{-1}\right)$ \\
\hline T00 & 1074 & 19,8049 & A & 140,6208 & A & $47,8190 \quad A$ & $15,2 \quad B$ & $13,9 \quad A$ & $0,0185 \quad B$ & $0,1309 \quad B$ \\
\hline D30 & 769 & 15,9932 & B & 113,2180 & B & $41,0772 \quad A$ & $16,2 A$ & $13,9 \quad A$ & $0,0208 \mathrm{~A}$ & $0,1471 \quad A$ \\
\hline D40 & 649 & 13,6289 & C & 96,9539 & C & $40,6912 \quad A$ & $16,3 \mathrm{~A}$ & $14,1 \quad \mathrm{~A}$ & $0,0211 \mathrm{~A}$ & $0,1498 \quad A$ \\
\hline D50 & 556 & 11,4861 & $\mathrm{D}$ & 82,1535 & D & $30,7054 \quad A$ & $16,2 \mathrm{~A}$ & $14,3 \mathrm{~A}$ & $0,0207 \mathrm{~A}$ & $0,1484 \quad A$ \\
\hline CV (\%) & 10,21 & 15,23 & & 12,00 & & 29,69 & 4,83 & 4,4 & 9,4 & 3,40 \\
\hline Erro padrão & 27 & 0,5977 & & 4,5926 & & 4,2061 & 0,2 & 0,2 & 0,0005 & 0,1917 \\
\hline
\end{tabular}

remanescentes de todas as intensidades testadas, e também da testemunha.

A competição pode ter iniciado aos quatro anos, mas se consolidou ao quinto ano pelo contato entre as copas, diminuindo a luz lateral individual. Provavelmente isso também ocorre no solo, com menor disponibilidade de água e nutrientes e afetam as árvores não tratadas pela plena ocupação do espaço de crescimento.

Dessa forma, o desbaste disponibilizou o espaço aéreo e subterrâneo para garantir um crescimento superior ao registrado para a testemunha, independente das intensidades utilizadas, até um ano após o tratamento.

O fechamento do dossel ocorreu no quinto ano, semelhante à observação de Pandey \& Brown (2000) que sugeriram que ela ocorre a partir dos quatro anos de idade. De outro lado, diferem de Kaosa-Ard (1998) que sugeriu, para povoamentos de espaçamento inicial menor, a aplicação do primeiro desbaste entre 5 a 10 anos de idade, podendo ser mecânico em sítios de boa qualidade.

Pérez \& Kanninen (2005) indicaram desbastes aos cinco anos, para sítios de qualidade média, nas intensidades de 40 a $45 \%$, dependendo do objetivo e da rotação planejada. E estes resultados indicam que o desbaste seletivo nas intensidades de 30 a 50\% em densidade pode ser aplicado em povoamentos de teca aos cinco anos de idade, implantados no espaçamento 3,0 x 3,0 m, em sítios semelhantes. Rondon (2006) não detectou diferenças na altura de teca, aos 76 meses de idade, ao estudar o efeito de espaçamentos de 3,0 x 2,0 m até 5,0 x 5,0 m, sem a aplicação de desbaste.

\section{CONCLUSÕES}

Um ano após a operação, intensidades de 20\%, 30\% e 40\% de desbaste seletivo aos quatro anos de idade em povoamento de teca 3,0 x 3,0 m não afetam o DAP, altura total e as médias individuais de área transversal e de volume, mas diminuem o ICA, em relação à testemunha. Aplicado aos cinco anos, nas intensidades de $30 \%$, 40\% e $50 \%$, aumenta o DAP e as médias individuais de área transversal e de volume, em relação à testemunha, mas não afeta a altura total e o ICA.

O primeiro desbaste seletivo pode ser aplicado aos cinco anos de idade, em povoamentos de Tectona grandis com condições semelhantes, com qualquer das intensidades testadas.

\section{AGRADECIMENTOS}

À empresa Teca do Brasil pelo apoio na realização deste trabalho.

\section{BIBLIOGRAFIA CITADA}

Briscoe, C. 1995. Silvicultura y manejo de teca, melina y pochote. CATIE, Informe Técnico No. 270, Turrialba, Costa Rica. $45 \mathrm{pp}$.

Caldeira, S.F.; Borges, S.A.S. 2004. Desarrollo de Azadirachta indica, Khaya ivorensis y Tectona grandis en Nossa Senhora do Livramento, MT, Brasil. In: $3^{\text {er }}$ Simposio Internacional sobre Manejo Sostenible de los Recursos Forestales. Memorias... Universidad de Pinar del Río, Pinar del Río, Cuba, 13pp. (CDRom).

Chaves, E.; Fonseca, W. 1991. Teca (Tectona grandis L.f.): especie de árbol de uso múltiple en América Central. CATIE, Informe Técnico No. 179, Turrialba. 47pp.

Cordero, L.D.F.; Kanninen, M. 2003. Hacia el manejo intensivo de la teca (Tectona grandis) en Centroamérica. 10pp. Disponível em www.una.ac.cr/inis/docs/teca/temas/PerezyKanninen1.pdf. Acesso em 17/02/05.

Ferreira, D.F. 2000. Análises estatísticas por meio do Sisvar para Windows versão 4.0. In: 45a Reunião Anual da Região Brasileira da Sociedade internacional de Biometria. Anais... UFSCar, São Carlos. p.255-258. Disponível em www.dex.ufla.br/-danielff/ softwares.htm. Acesso em 27/03/05.

Figueiredo, E.O. 2001. Reflorestamento com teca (Tectona grandis L.F.) no estado do Acre. EMBRAPA, Documento No. 65, Rio Branco. 28pp.

Galloway, G.; Ugalde, L.; Vásquez, W. 2001. Importance of density reductions in tropical plantations: Experiences in Central America. Forests, Trees and Livelihoods. 11(3): 217-232. 
Jerez, M.; Vicent, L.; Moret, Y.; Gonzáles, R. 2003. Regímenes de espaciamiento inicial y aclareo en plantaciones de Teca (Tectona grandis L.f.) en Venezuela. 16pp. Disponível em www.una.ac.cr/ inis/docs/teca/temas/RegmenesdEspaciamiento1.pdf. Acesso em 27/10/05.

Kaosa-Ard, A. 1998. Overview of problems in teak plantation establishment. In: Teak for the future. Second Regional Seminar on Teak. FAO, 1995. Proceedings... Teaknet, (1): 49-59.

Krishnapillay, B. 2000. Silvicultura y ordenación de plantaciones de teca. Unasylva, 51(201): 1-13.

Matricardi, W.A.T. 1989. Efeitos dos fatores do solo sobre o desenvolvimento da teca (Tectona grandis L.F.) cultivada na Grande Cáceres - Mato Grosso. Dissertação de Mestrado, USP, Escola Superior de Agricultura Luiz de Queiroz, Piracicaba. 135pp.

Pandey, D.; Brown, C. 2000. La teca: una visión global. Unasylva, 51(201): 1-18.
Perez, D.; Kanninen, M. 2005. Stand growth scenarios for Tectona grandis plantations in Costa Rica. Forest Ecology and Management, 210(1-3): 425-441.

Rondon, E.V. 2006. Estudo de biomassa de Tectona grandis L.f. sob diferentes espaçamentos no estado de Mato Grosso. Revista Arvore, 30(3): 337-341.

Salas, E.C.; Gonzáles, W.F. 2003. Ensayos de aclareo y crecimiento en plantaciones de teca (Tectona grandis, L.f.) en la Península de Nicoya, Costa Rica. 17pp. Disponível em www.una.ac.cr/inis/ docs/teca/temas/RAenTECA2.pdf. Acesso em 25/10/05.

Vincent, L.; Moret, A.Y.; Jerez, M. 2000. Comparación de algunos regímenes de espesura en plantaciones de teca en el área experimental de la Reserva Forestal de Caparo, Venezuela. Rev. Forestal Venezolana, 44(2): 87-95.

Recebido em 15/06/2007

Aceito em 13/02/2008 
